\title{
Latexfreie Kondome
}

Frage:

Die Latexallergie nimmt deutlich zu. Oft sind jüngere Menschen betroffen. Die Frage nach latexfreien Kondomen wird öfter gestellt. Eigene Nachforschungen blieben ergebnislos. Sind latexfreie Kondome im Handel?

\section{Antwort:}

Latexfreie Kondome sind derzeit in Deutschland nicht im Handel und werden nach Auskunft der Hersteller wahrscheinlich fühestens in einem $1 / 2$ Jahr auf dem deutschen Markt erhältlich sein.

In den USA ist seit kurzem ein latexfreies Kondom aus Polyurethan mit dem Fabrikatnamen „Avanti“ im Handel. Der Hersteller ist: London International U.S. Holdings, Inc. Schmid Laboratories Division, Sarasota, FL 34230-4703 Duron.

Priv.-Doz. Dr. A. Heese

Universitäts-Hautklinik

Hartmannstraße 14

D-91052 Erlangen

\section{Sonnenbedingte Hautreizung nach Tretinoinbehandlung}

Frage:

Eine Patientin wurde wegen einer Akne vulgaris mit einer Tretinoincreme behandelt. Diese wurde gut vertragen. Die Patientin berichtete, sie habe 3 Wochen nach Beendigung der Behandlung einen Urlaub in Südeuropa angetreten und dort Hautreizungen im Gesicht bekommen, die sie früher nicht gehabt ha-

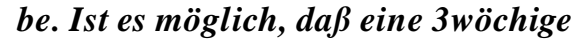
Latenz zwischen Behandlung und intensiver Sonneneinstrahlung nicht ausreicht, um nach einer Tretinoinbehandlung vor derartigen Reizen sicher zu sein?

\section{Antwort:}

Durch die Behandlung mit Tretinoin (Vitamin-A-Säure) kommt es zu einer Verdünnung der Hornschicht, einer gesteigerten transepidermalen Wasserabgabe, einer geringen Pigmentaufhellung und einer leichteren Irritabilität der Haut auf Hautirritantien.

Fast alle dieser Phänomene werden durch die gesteigerte Proliferation der Epidermis und die beschleunigte Abschilferung der Korneozyten und das damit bedingte dünne Stratum corneum erklärt.
Unter einer Tretinoinbehandlung sollte eine vorsichtige Sonnenexposition erfolgen, da wie bei verschiedenen Schälbehandlungen leichter ein Sonnenbrand auftreten kann.

Drei Wochen nach Absetzen der Schälbehandlung mit Tretinoin ist jedoch das Stratum corneum wieder voll funktionsfähig aufgebaut. Daher sind die von der Patientin angegebene Hautreizungen, die 3 Wochen nach Beendigung der Tretinoinbehandlung nach einem Urlaub in Südeuropa nicht in Verbindung mit dem Medikament zu sehen. Allerdings sollte aus der Anamnese erfragt werden, ob wegen der intensiven Sonneneinstrahlung andere UV-bedingte Hautveränderungen aufgetreten sind, wie beispielsweise eine polymorphe Lichtdermatose. Wurden Lichtschutzmittel oder sonstige andere Hautpflegeprodukte benutzt? Auch auf eine Hautreizung durch solche Produkte wäre zu achten.

Prof. Dr. G. Plewig

Dermatologische Klinik und Poliklinik der Universität

Frauenlobstraße 9-11

D-80337 München 